\title{
Iris Pattern Positioning with Preserved Edge Detector and Overlay Matching
}

\author{
Kwang Ryol Ryu, Member, KIMICS
}

\begin{abstract}
An iris image pattern positioning with preserved edge detector, ring zone and clock zone, frequency distribution and overlay matching is presented in this paper. Edge detector is required to be powerful and detail. That is proposed by overlaying Canny with LOG (CLOG). The two reference patterns are made from allocating each gray level on the clock zone and ring zone respectively. The normalized target image is overlaid with the clock zone reference pattern and the ring zone pattern to extract overlapped number, and make a matched frequency distribution to look through a symptom and position of human organ and tissue. The iterating experiments result in the ring and clock zone positioning evaluation.
\end{abstract}

Index Terms - Iris pattern positioning, overlay matching, preserved edge detector, CLOG, frequency distribution, ring zone and clock zone.

\section{INTRODUCTION}

An iris image pattern is expanding more such as medical, law enforcement, human identification and verification, the handicapped and surveillance in digital image processing. The medical of them is applied to diagnose human health. Iris image pattern gives a hint relating to human organs in iridology. Iridology were discovered and used successfully is now being understood. The eyes are normally reserved for biological analysis. There are about ninety different organs and tissue represented in the eyes. The eye patterns are specific for each individual that are believed to be far more accurate and permanent than even fingerprints. Through the various patterns and colors that appear in the iris, it is possible to detect underactive organs or tissues and overactive organs and tissues because the eyes show the problem [1-4]. The characteristics of iris pattern are extracted by edge detector. There are so many detecting algorithms. The most powerful algorithm is known as Canny and Laplacian of Gaussian (LOG). The Canny may preserves edges to extract a characteristic in comparison with LOG but not detail [5-8].

This paper proposes a matching algorithm between the

\footnotetext{
Manuscript received May 25, 2010; revised May 31, 2010; accepted June 7, 2010.

Kwang Ryol Ryu is with Department of Electronic Engineering, Mokwon University, Daejeon, 302-729, S. Korea (Tel:+82-42-829-7651, Email: conan@mwu.ac.kr).
}

reference iris pattern and a new iris pattern. The new edge detection is proposed by overlaying Canny and LOG with threshold. The reference image pattern is made of clock zones 0 to 14 and the ring zones 0 to 8 separation allocated each gray level included extra area with respect to each organs. The normalized target pattern is overlaid by the reference pattern, and calculates the logical operation to take overlapped numbers. An organ problem is estimated by frequency distribution of the overlay matching.

\section{EDGE DETECTOR AND OVERLAY MATCHING}

\section{A. Preserved Edge Detector}

Iris image pattern is formed by using edge detection of iris. A color image is converted to gray image. Edge detection is required to be powerful and less susceptible to noise and to preserve edges. The Canny and Laplacian of Gaussian (CLOG) detectors enable to lead that purpose. The Canny method is overlaid by the Laplacian of Gaussian method to be preserved each other. The Canny detector is the most powerful edge detection and more likely to detect true weak edges that uses two thresholds to detect strong and weak edges, and includes the weak edges in the output only if they are connected to strong edges. That may result in a powerful detector to find iris pattern. The implementation of the detector requires the following steps. That finds edges by looking for local maxima of the gradient of an iris image. The gradient is calculated using the derivative of a Gaussian filter with a specified standard deviation to reduce noise. The local gradient and edge detection are computed at each point. An edge point is defined to be a point which of strength is locally maximum in the direction of the gradient. It gives rise to ridges in the gradient magnitude image. The ridge pixels are thresholded using two thresholds. The first element is the low threshold, and the second element is the high threshold. Finally the method performs edge linking by incorporating the weak pixels.

Laplacian of Gaussian is a second order derivative edge detection that is less susceptible to noise, and is powerful zero crossing detector. It is defined as equation (1) in $\mathrm{m} \mathrm{x}$ $n$ image.

$$
h(m, n)=\frac{1}{\pi \sigma^{4}}\left[1-\frac{\left(m^{2}+n^{2}\right)}{2 \sigma^{2}}\right] e^{\frac{-\left(m^{2}+n^{2}\right)}{2 \sigma^{2}}}
$$


The wider the function $\sigma$, the wider the edge that will be detected, and narrow function will detect sharp edges and more detail. The first zero crossing is at $\sqrt{2 \sigma}$. The greater of $\sigma$, the wider the convolution mask necessary. A discrete approximation of Laplacian of Gaussian function with Gaussian $\sigma=1.4$ and $\sigma=1.2$ are shown in Fig. 1 as convolution masks 9x9.

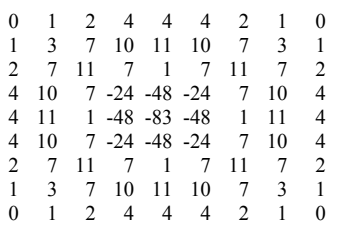

(a) $\sigma=1.4$

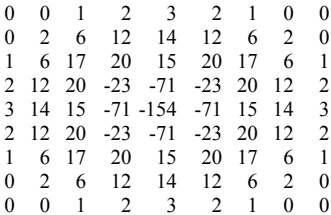

(b) $\sigma=1.2$
Fig. 1 Convolution masks

The preserved edge detector, CLOG comes from overlaying Canny edged image into Laplacian of Gaussian edged image with adding, averaging and thresholding as shown in Fig. 2.

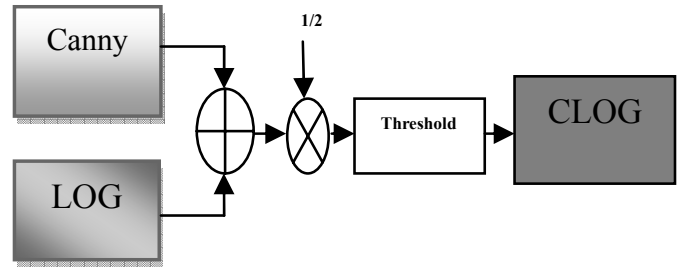

Fig. 2 Overlaid edge detector blocks

\section{B. Iris Reference Patterns}

The research of the significance of the whole eye included the retina, sclera and sclera vessels, iris, palpabrae, and even the cornuncle, for each of the structure is unique. The iridology chart of the right eye may be separated in shaping of clocks and rings intuitively as shown in Fig. 3. [1]

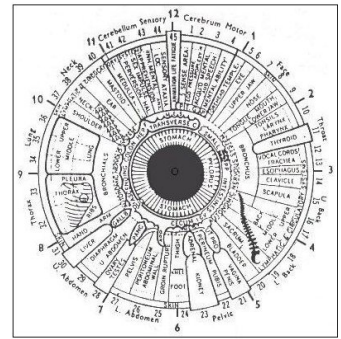

Fig. 3 Iridology chart

The sections of eye are pupil, iris and sclera composed of pattern. The iris is composed of three layers from anterior to posterior; anterior border layer, stromal layer and posterior epithelium layer. The anterior border layer is a modification of the middle stromal layer. It is composed of two layers: an anterior fibroblastic layer and a posterior pigmented layer. The posterior epithelium layer is heavily pigmented with black and brown granules. The iris develops neurologically from the pupil outward. There are a total of seven concentric zones of the right iris. Each ring zone contains certain organ and tissue roughly. The ring zones are 7 separate zones. Zone 1: gastric mucosa, zone 2: small and large intestinal tracts, zone 3: heart, pancreas, pituitary gland, adrenal glands, aorta, gall bladder, solar plexus, parathyroid, uterus and prostate pineal, zone 4: bronchial tubes, zone 5: brain and reproductive organs, zone: 6 spleen, thyroid, liver, kidneys and spine, zone 7: lineated into a superior and inferior section, and zone 8: sclera. The inferior contains lymphatic and circulatory systems, motor and sensory nerves. The superior aspect reflects changes in the sweat glands and skin as shown in Fig. 4 (a).

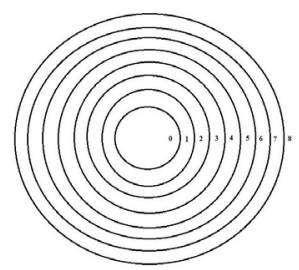

(a)Ring zone

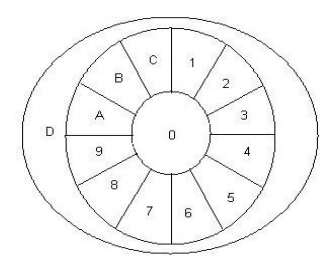

(b) Clock zone
Fig. 4 Iris separated zones

The clock zones are roughly zone 1: cerebrum, zone 2 : eye, nose, temple and forehead, zone 3: tonsils, pharynx and larynx, zone 4: scapula and dorsal muscles, zone 5: bladder vagina, zone 6: foot and kidney, zone 7: knee, zone 8: spleen sciatic, zone 9: ribs pleura, zone A: heart and lungs, zone $\mathrm{B}$ : ears, zone $\mathrm{C}$ : cerebellum, zone D: sclera, and otherwise cilia E. The iris enables to be laid out neurologically like a 12 times clock zones as shown in Fig. 4 (b).

\section{Pattern Overlay Matching}

The normalized size clock zone reference pattern is allocated to each number from 0 to 14 included pupil, sclera and extra zone corresponding to gray level intensity in Fig. 5 and Table 1. The target iris pattern is made from the edge detector on input iris image. The edged image pixel value is changed by threshold, that is, if a pixel is great than threshold then 255 else 0 . This target pattern is overlaid with the clock zone reference pattern, and calculate logical operation each other, if a changed pixel is equal to 1 then the pixel is replaced with the clock zone pixel to match out the pixel values of a reference zone, which forms distribution of frequency to each zones. Finally the maximum frequency will be implied the organ or tissue.

The ring zone matching process is similar to clock zone matching. The ring zone reference of the normalized size pattern is given the number from 0 to 8 to each ring like the clock zone reference in Fig. 6 and Table 2. 
TABLE 1

CLOCK ZONE PIXEL VALUES

\begin{tabular}{|l|l|l|l|l|l|l|l|l|l|l|l|l|l|l|l|}
\hline $\begin{array}{l}\text { clock } \\
\text { zone }\end{array}$ & C0 & C1 & C2 & C3 & C4 & C5 & C6 & C7 & C8 & C9 & C10 & C11 & C12 & C13 & C14 \\
\hline pixel & 10 & 25 & 40 & 55 & 70 & 85 & 100 & 115 & 130 & 145 & 160 & 175 & 190 & 220 & 240 \\
\hline
\end{tabular}

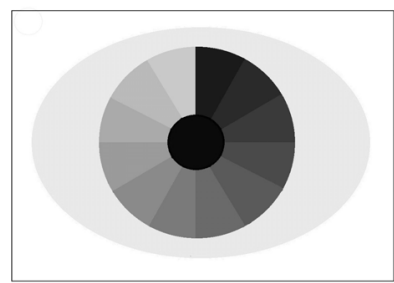

Fig.5 Iris reference pattern for clock zone

A target iris is overlaid with the ring zone reference pattern, and if a changed pixel is equal to 1 then the pixel is replaced with the ring zone pixel. The matched ring zone is found out the frequency distribution. Finally the results of the clock zone and ring zone enable to extract the positions of angles and rings to be corresponded to an organ or tissue.

TABLE 2

RING ZONE PIXEL VALUES

\begin{tabular}{|c|c|c|c|c|c|c|c|c|c|}
\hline ring zone & R0 & R1 & R2 & R3 & R4 & R5 & R6 & R7 & R8 \\
\hline pixel & 15 & 35 & 50 & 75 & 90 & 110 & 135 & 150 & 195 \\
\hline
\end{tabular}

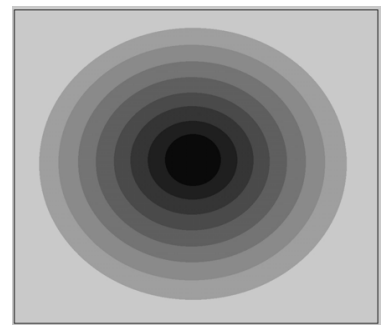

Fig.6 Iris reference pattern for ring zone

\section{EXPERIMENT AND RESULTS}

The pattern sizes for the overlay edge detecting image and ring and clock reference pattern for overlay matching are gray images $480 \times 320$ to extract the positions for the clocks angle and radii interval to estimate a organ and tissue. The edged image depends on the threshold quite and standard deviation. The standard deviation takes 1.2 or 1.4 , the threshold 0.05 to 0.1 , and convolution mask $9 \times 9$. The original image Fig. 7 (a) is transferred with Canny edged image Fig. 7 (b) and LOG edged image Fig. 7 (c) for the overlaid edged CLOG Fig. 7 (d). CLOG edged image is obtained by averaging Canny edge and LOG edge. An edged image threshold takes 10 to convert to BW image.

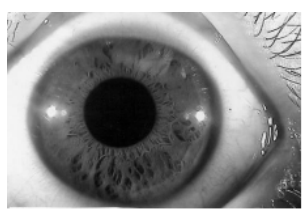

(a)Original

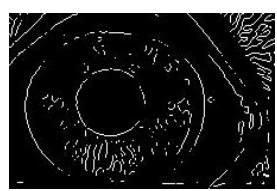

(c) $\mathrm{LOG}$

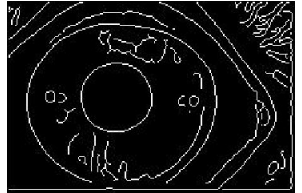

(b)Canny

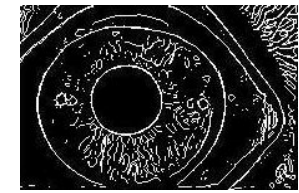

(d)CLOG
Fig. 7 Target iris and edged images

The frequency distribution is given in the overlay matching between the reference pattern and the target edged image pattern as shown in Fig. 5, Fig. 6 and Fig. 7. The clock zone frequency distribution shows high at zone 7 and 12, and sclera 13 and cilia 14 higher in Fig. 8. A target image includes reflection of light to be error at zone 3 and 9. The zone 5 indicates bladder vagina to be something wrong, and zone 7 , knee. The ring zone frequency distribution indicates high at zone 6 and 7 as shown in Fig. 9. The zone 6 shows spleen, thyroid, liver, kidneys and spine to be symptom, and zone 7 is lineated into a superior and inferior section.

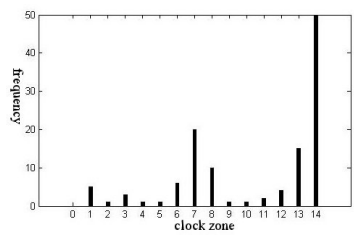

Fig. 8 Clock zones frequency distribution

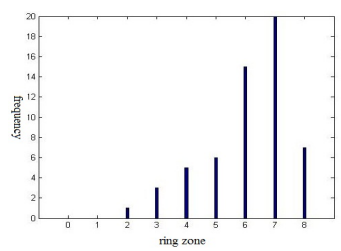

Fig. 9 Ring zone frequency distribution

It is hard to discriminate a ring and clock zone to an extreme. The iterative experiment results in the positioning accuracy about $62 \%$ in the ring zone and $65 \%$ in the clock zone at the average.

Especially the ring 1and 2 pattern are separated to be the part of surrounding pupil R1 and ANW (Autonomic Nerve Wreath) R2 (sawtooth-line shaped) as shown in Fig. 10. The lining of the pupil has been found to be helpful in detecting metabolic and pathological problems. It has been noted that the papillary lining can become irregular when the patient is having a glucose tolerance problem, especially diabetes mellitus. The autonomic nerve wreath 
is also significant as a landmark. It surrounds the gastrointestinal tract and gives an outline of the stomach, small intestines and large intestine at all times, the indication of a prolapsed and even a spastic colon because of the location of wreath.

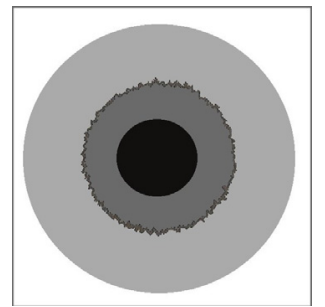

Fig. 10 Pupil and ANW reference pattern

TABLE 3

PATTERN LOOKUP TABLE

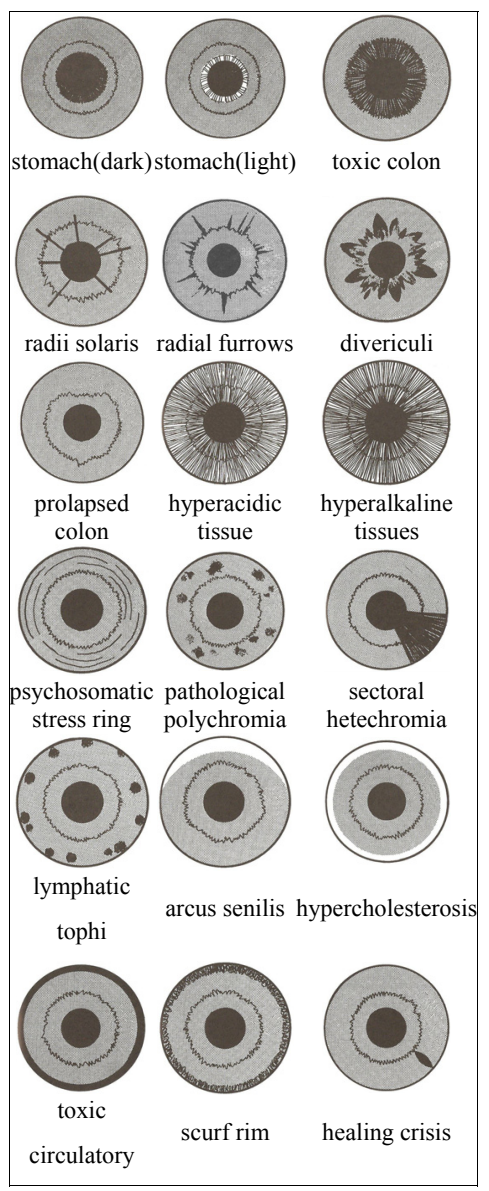

An input image is normalized by $640 \times 480$ with centered pupil size. The significant patterns are discriminated by the Lookup table in the table 3 to be overlaid with the pupil and ANW reference pattern. An input pattern compares with 18 patterns in the lookup table and the frequency distribution shows about the clocks and ring zones. The success matching rates to the clock and ring are 60 and $69 \%$ at the average respectively.

\section{CONCLUSIONS}

A method of the positioning of iris image pattern with preserved edge detector and overlay matching is presented in this paper. The edged image depends on the threshold quite, standard deviation and convolution mask. The positioning of iris pattern is taken by the clock zone and ring zone. The matching is given in the two reference pattern and a target image pattern to overlay each other respectively. That makes the frequency distribution to estimate a symptom position. This method accuracy depends on an edge detector and reducing noise. An edge detector needs new for accuracy. The positioning enables to estimate a symptom roughly. However it is hard to be shown and discriminated on a whole shapes like scurf rim, stress ring, radii solaris-radiate, lipid ring, and etc surrounding the autonomic nerve wreath and pupil to diagnose an organ and tissue. The significant iridology findings require a new technique and also color change discrimination.

\section{REFERENCES}

[1] Donald R. Bamer, Practical Iridology and Sclerology, Woodland Publishing, 1996.

[2] K.R. Ryu, "Area Recognition for Iries Diagnosis with Edge Image Pattern Matching", Proceedings of KIMICS, vol5, no.1, pp.653$655,2001$.

[3] K. R. Ryu and D. H. Chai, "Moving Iris Tracking and Screen Cursor Real Time Matching", J. of KIMICS, vol.9, no.7, pp.15081512,2005

[4] Christelloic Tisse, Lionel Martin, Lionel Torres and Michel Robert, "Person Identification Technique using Human Iris Recognition".

[5] John Canny, "A Computational Approach for Edge Detection", IEEE Trans. Pattern Anal. Machie Intell., vol.8, no.6, pp.679-698, 1986.

[6] Andres Hertus and Gerard Medioni, "Detection of Intensity Changes with Subpixel Accuracy using Laplacian Masks", IEEE Transaction on Pattern Anaysis and Machine Intelligence vol. no.5, PAMI-8, pp.651-665, September 1986.

[7] Rafael C. Gonzalez and Richard E. Woods, Digital Image Processing, pp. 693-696, Prentice Hall, 2002.

[8] Anil K. Jain, Fundamental of Digital Image Processing, Prentice Hall, 1989.

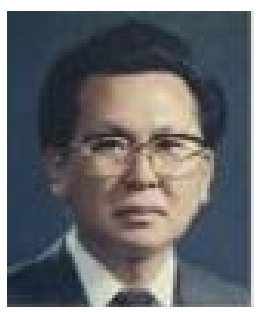

\section{Kwang Ryol Ryu}

Received the PhD. Degree from Kyunghee University in 1988, and is currently a professor at the Dept. of Electronic Engineering at Mokwon University, worked as a visiting Professor at the Dept. of Neurological Surgery at University of Pittsburgh Medical Center in USA in 2006 2008. His areas of research include Digital Signal Processing, Imaging and Vision, and Bio-Medical Electronics. 